\title{
Follow-up of Patients with Atypical Lymphoproliferation in Bone Marrow or Lymph Node Biopsy
}

Rafiye Ciftciler', [MD]

ORCID: 0000-0001-5687-8531

Ali Erdinc Ciftciler', [MD]

ORCID: 0000-0001-7612-2707

Emine Arzu Saglam ${ }^{3}$, [MD]

ORCID: 0000-0002-0076-8293

Yahya Buyukasık', [MD]

ORCID: 0000-0002-4764-2348

${ }^{1}$ Hacettepe University Faculty of Medicine, Department of Hematology, Ankara, Turkey.

${ }^{2}$ Ortakoy State Hospital, Department of General Surgery, Aksaray, Turkey.

${ }^{3}$ Hacettepe University Faculty of Medicine, Department of Pathology, Ankara, Turkey.

Corresponding Author: Rafiye Ciftciler Departments of Hematology, Hacettepe University

Faculty of Medicine, Ankara, Turkey.

Phone: +90 03123053050

E-mail: rafiyesarigul@gmail.com

https://doi.org/10.32552/2021.ActaMedica.520

\section{w ABSTRACT Con}

Objective: Patients presenting with various complaints to the hematology polyclinic may initially be diagnosed with an atypical lymphoproliferation in bone marrow or lymph node biopsy. The aim of this study was to determine whether a hematological disease, immunodeficiency syndrome, or other diseases were diagnosed during follow-up of patients with an initial diagnosis of atypical lymphoproliferation in bone marrow or in lymph node biopsy.

Materials and Methods: Adult ( $\geq 18$ years) patients who were admitted to the Hacettepe University Hospital, for various symptoms between 2002 and 2018 were searched for in our hematology department electronical database.

Results: A total of 52 patients were found with atypical lymphoproliferation in lymph node or bone marrow biopsy. The patients had been followed for a median of 9.2 months (0.03-86.2). Hematological neoplasia developed in $32(61.6 \%)$ of the 52 patients and primary immunodeficiency was detected in $6(11.5 \%)$ of the 52 patients. Twentysix patients $(50 \%)$ were diagnosed Non-Hodgkin lymphoma during follow up, 1 patient (1\%) was diagnosed chronic lymphocytic leukemia, 5 patients (9.6\%) were diagnosed Hodgkin lymphoma and 6 patients (11.5\%) were diagnosed primary immunodeficiency. Median time was 2.3 months (0.2-25 months) between atypical lymphoproliferation report in bone marrow or lymph node biopsy and the diagnosis of patients.

Conclusion:Inconclusion, patientswhohaveatypicallymphoproliferation in the lymph node or bone marrow biopsy should be followed up in the hematology outpatient clinic. Because, during follow-up, diseases such as hematological neoplasia or immunodeficiency can be diagnosed in patients with atypical lymphoproliferation.

Keywords: Atypical lymphoproliferation, non-hodgkin lymphoma, immunodeficiency

Received: 19 October 2020, Accepted: 3 January 2021,

Published online: 5 February 2021

\section{INTRODUCTION}

Atypical lymphoproliferation (ALP) is not a pathological or clinical definition. However, ALP indicates cases where the pathologist revealed the concern about an abnormal process but where findings fell short of diagnosis of a specific reactive or neoplastic entity [1]. ALP encompasses characteristics of reactive polymorphous proliferations to clonal malignant diseases, they are often difficult to diagnose and treat effectively. There is no definition for classifying ALP that predicts whether a patient will have a self-limited illness or one that will finally result in lymphoma or death [2]. Atypical lymphoid proliferation or hyperplasia is defined as damaged or effaced structure of the lymph nodes, however the histology does not meet the criteria for malignancy. ALP may be a 
manifestation of damage to the immune system and may progress to malignancy [2]. In most cases, ALP are associated with an abnormal immune response to some stimulant. For correct diagnosis, careful evaluation is needed with immunohistologic, karyotypic, virologic and genotypic analyses and the clinical findings, previous medications and family history of the patients.

Bone marrow or lymph node biopsy is reported as atypical lymphoproliferation in patients presenting with various complaints to hematology polyclinic. The aim of this study was to determine whether hematological disease, immunodeficiency syndrome, or other diseases were diagnosed in patients with atypical lymphoproliferation in bone marrow or in lymph node biopsy.

\section{MATERIALS and METHODS}

\section{Study Design and Data Collection}

Adult ( $\geq 18$ years) patients who were admitted to the Hacettepe University Hospital, for various symptoms between 2002 and 2018 were searched within our hematology department electronical database. This database is used for routine patient records and it is maintained prospectively. Details of pathology results were obtained from the hospital database. Patients with obvious clinical abnormalities related to a lymphohematopoietic disorder were excluded. Lymph node or bone marrow biopsy was performed according to patient symptoms. A total of 52 cases were found. The cases included in this study were ones who were not able to diagnosed either as benign or malignant with bone marrow or lymph node biopsy at the time of admission to the hematology clinic. The patients were evaluated for excisional or fine needle aspiration of lymph node biopsies. Immunohistochemical examination of lymph node or bone marrow biopsy was evaluated. Immunophenotyping studies by flow cytometry were evaluated. Bone marrow or lymph node biopsies of these patients were reported as atypical lymphoproliferation. All of the ethical considerations had been strictly followed in accordance with the 1964 Helsinki declaration. As a standard care/action of the hospitals of the Hacettepe Medical School, it has been recognized from the patient records that all of the studied patients had given informed consents at the time of hospitalization and before the administration of relevant diagnostic/therapeutic standard of care.

\section{Statistical Analyses}

Demographic characteristics were presented using proportions and medians (minimum-maximum) for categorical and continuous variables, respectively. Statistical comparisons were made using Chisquare for categorical data. Student t-test (for two independent samples) or Kruskal Wallis test (for more than two independent samples) were used for comparison of continuous numerical data. The statistical analyses were performed using SPSS v17 (SPSS Inc, Chicago, IL).

\section{RESULTS}

\section{Characteristics of the Study Population}

A total of 52 patients were found with ALP in lymph node or bone marrow biopsy. Patient characteristics are summarized in Table 1. There were 30 (57.7\%) males and 22 (42.3\%) females with a median age of 50 (range, 18-80) years at the time of presentation to the Hematology clinic who had underwent bone marrow or lymph node biopsy. Twenty-seven (51.9\%) patients underwent bone marrow biopsy and 25 patients (48.1\%) underwent lymph node biopsy for their complaints. Nine patients (36\%) underwent fine needle aspiration biopsy and 16 patients (64\%) underwent excisional lymph node biopsy. The lymph node biopsy was performed most frequently from the cervical, axillary and inguinal areas. The biopsies were performed because the patients had complaints of B symptoms (fever, loss of gain and night swats) (17 patients, 32.7\%), lymphadenopathy (17 patients, 32.7\%), cytopenia (16 patients, 30.8\%) and hepatosplenomegaly (2 patients, 3.8\%). Most of patients had no known comorbidities at the time of presentation (32 patients, 61.6\%). Other patients had co-morbidities such as hypertension (8 patients, $15.3 \%)$, diabetes mellitus (5 patients, 9.6\%), coroner artery disease (3 patients, 5.7\%) and hypothyroidism (4 patients, 7.6\%). Twentyfour patients (46.1\%) had flow cytometry results. Immunohistochemistry in bone marrow or lymph node biopsy was available in 49 (94.2\%) of patients. However, immunohistochemistry and flow cytometry could not provide clear hematologic disease in those patients. Clonality was performed 
on the lymph node and bone marrow biopsies of these patients. However, clonality could not be shown. Additionally, there were no clear indications of a reactive lymph node. These modalities failed to confirm a benign or malignant process at initial admission at the hospital.

\section{Prevalence of Hematological or Other Disorders}

The patients had been followed for a median of 9.2 months (0.03-86.2). Thirty-eight (73.1\%) patients were diagnosed with a hematological malignancy or primary immunodeficiency. Twenty-six patients (50\%) were diagnosed $\mathrm{NHL}$ in the follow up, 1 patient (1\%) was diagnosed as chronic lymphocytic leukemia, 5 patients (9.6\%) were diagnosed Hodgkin lymphoma and 6 patients (11.5\%) were diagnosed primary immunodeficiency by immunologists. Fourteen patients (26.9\%) were not diagnosed with any hematological disease and they are still being followed up in the hematology outpatient clinic with no significant complications. Median time was 2.3 months (0.2-25 months) between ALP report in bone marrow or lymph node biopsy and the diagnosis of patients as shown in Figure 2. Photomicroscopic images of the patient who had ALP on lymph node biopsy are shown in Figures 3 and 4.

Table 1. Demographic and clinical characteristics of patients

\begin{tabular}{|c|c|}
\hline Variable & $\mathbf{N}(\%)$ \\
\hline Patients & 52 \\
\hline Age (range) years & $50(18-80)$ \\
\hline Male/female & $30 / 22(57.7 \% / 42.3 \%)$ \\
\hline \multicolumn{2}{|l|}{ Complaints } \\
\hline B symptoms & $17(32.7 \%)$ \\
\hline Lymphadenopathy & $17(32.7 \%)$ \\
\hline Hepatosplenomegaly & $2(3.8 \%)$ \\
\hline Cytopenia & $16(30.8 \%)$ \\
\hline \multicolumn{2}{|l|}{ Atypical lymphoproliferation (n, \%) } \\
\hline Lymphadenopathy & $25(48.1 \%)$ \\
\hline Fine needle aspiration biopsy & $9(36 \%)$ \\
\hline Excisional biopsy & $16(64 \%)$ \\
\hline Bone marrow & $27(51.9 \%)$ \\
\hline Flow cytometry analyses & $24(46.1 \%)$ \\
\hline Immunohistochemistry in bone marrow or lymph node biopsy & $49(94.2 \%)$ \\
\hline \multicolumn{2}{|l|}{ The tissue that hematological malignancy was diagnosed } \\
\hline Bone marrow & $6(18.8 \%)$ \\
\hline Lymphadenopathy & $22(68.8 \%)$ \\
\hline Spleen & $4(12.4 \%)$ \\
\hline \multicolumn{2}{|l|}{ Diagnoses (n, \%) } \\
\hline Diffuse large B cell lymphoma & $14(26.9 \%)$ \\
\hline Low grade B cell lymphoma & $2(3.8 \%)$ \\
\hline Mantle cell lymphoma & $1(1.9 \%)$ \\
\hline Peripheral T cell lymphoma & $9(17.3 \%)$ \\
\hline Chronic lymphocytic leukemia & $1(1.9 \%)$ \\
\hline Hodgkin lymphoma & $5(9.6 \%)$ \\
\hline Primary immunodeficiency syndrome & $6(11.5 \%)$ \\
\hline Patients who are still being followed up & $14(26.9 \%)$ \\
\hline \multicolumn{2}{|l|}{ Treatment regimen $(n, \%)$} \\
\hline R-CHOP (rituximab, cyclophosphamide, doxorubicin, vincristine, prednisolone) & $15(28.1 \%)$ \\
\hline CHOEP (cyclophosphamide, doxorubicin, vincristine, etoposide, prednisolone) & $9(17.3 \%)$ \\
\hline FCR (fludarabine, cyclophosphamide, rituximab) & $1(1.9 \%)$ \\
\hline ABVD (doxorubicin, bleomycin, vinblastine, dacarbazine) & $5(9.6 \%)$ \\
\hline IVIG (intravenous immunoglobulin) & $6(11.5 \%)$ \\
\hline No treatment (Patients who are being followed up) & $14(26.9 \%)$ \\
\hline
\end{tabular}




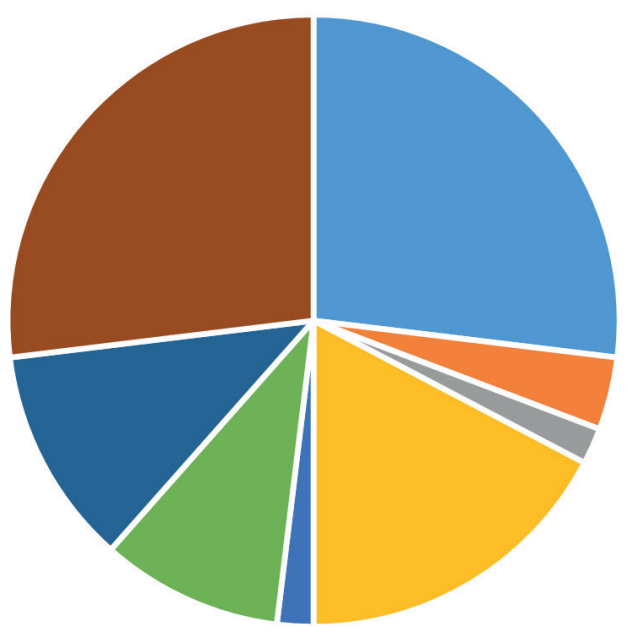

- Diffuse large B cell lymphoma (26.9\%)

- Low grade B cell lymphoma (3.8\%)

- Mantle cell lymphoma(1.9\%)

- Peripheral T cell lymphoma (17.3\%)

- Chronic lymphocytic leukemia (1.9\%)

- Hodgkin lymphoma (9.6\%)

- Primary immunodeficiency syndrome (11.5\%) - Patients who are in the followed up (26.9\%)

Figure 1. The diagnosis of the patients during follow-up who had atypical lymphoproliferation in initial bone marrow or lymph node biopsy

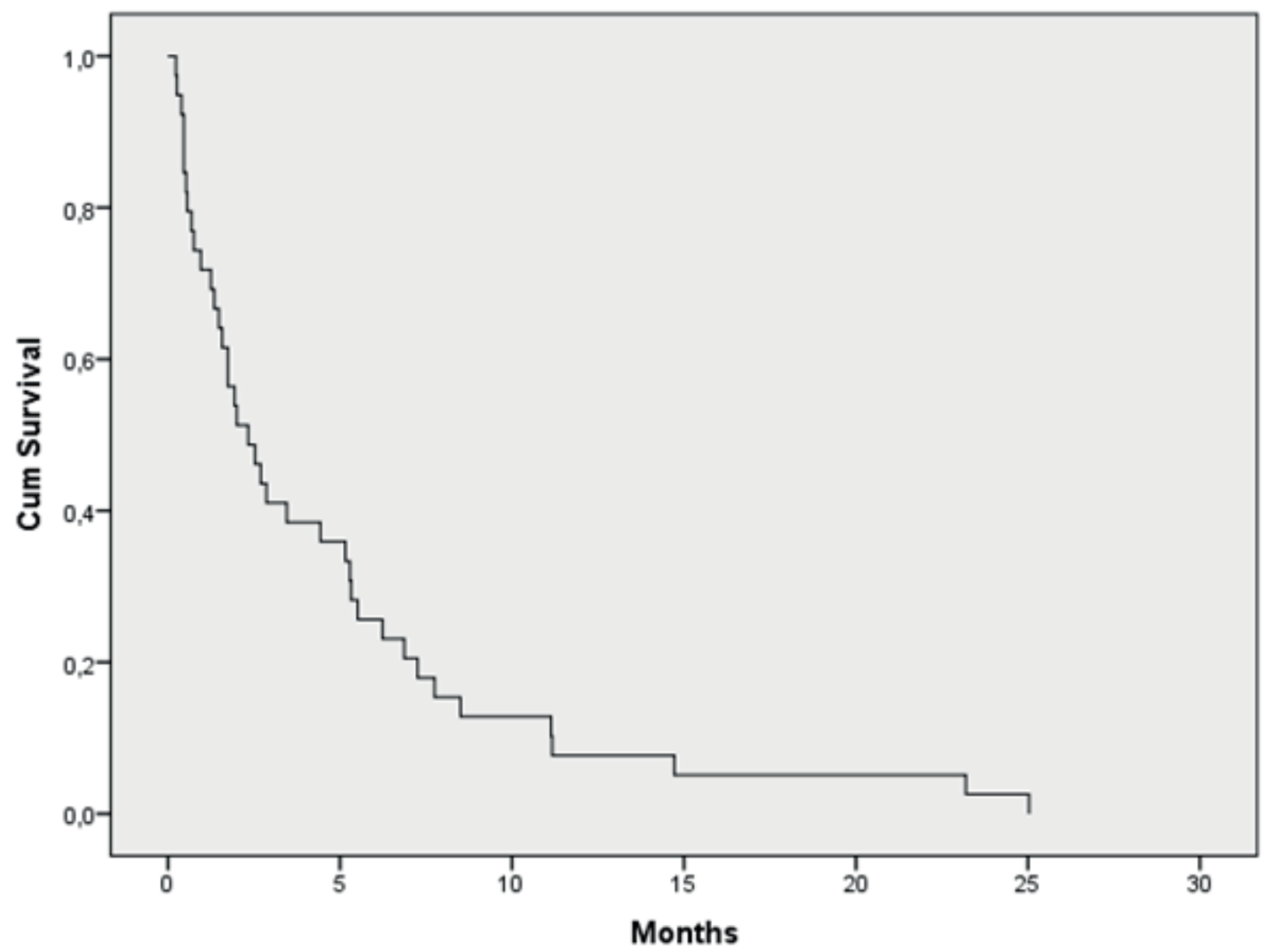

Figure 2. Transformation free survival of patients with atypical lymphoproliferation in bone marrow or lymph node biopsy 


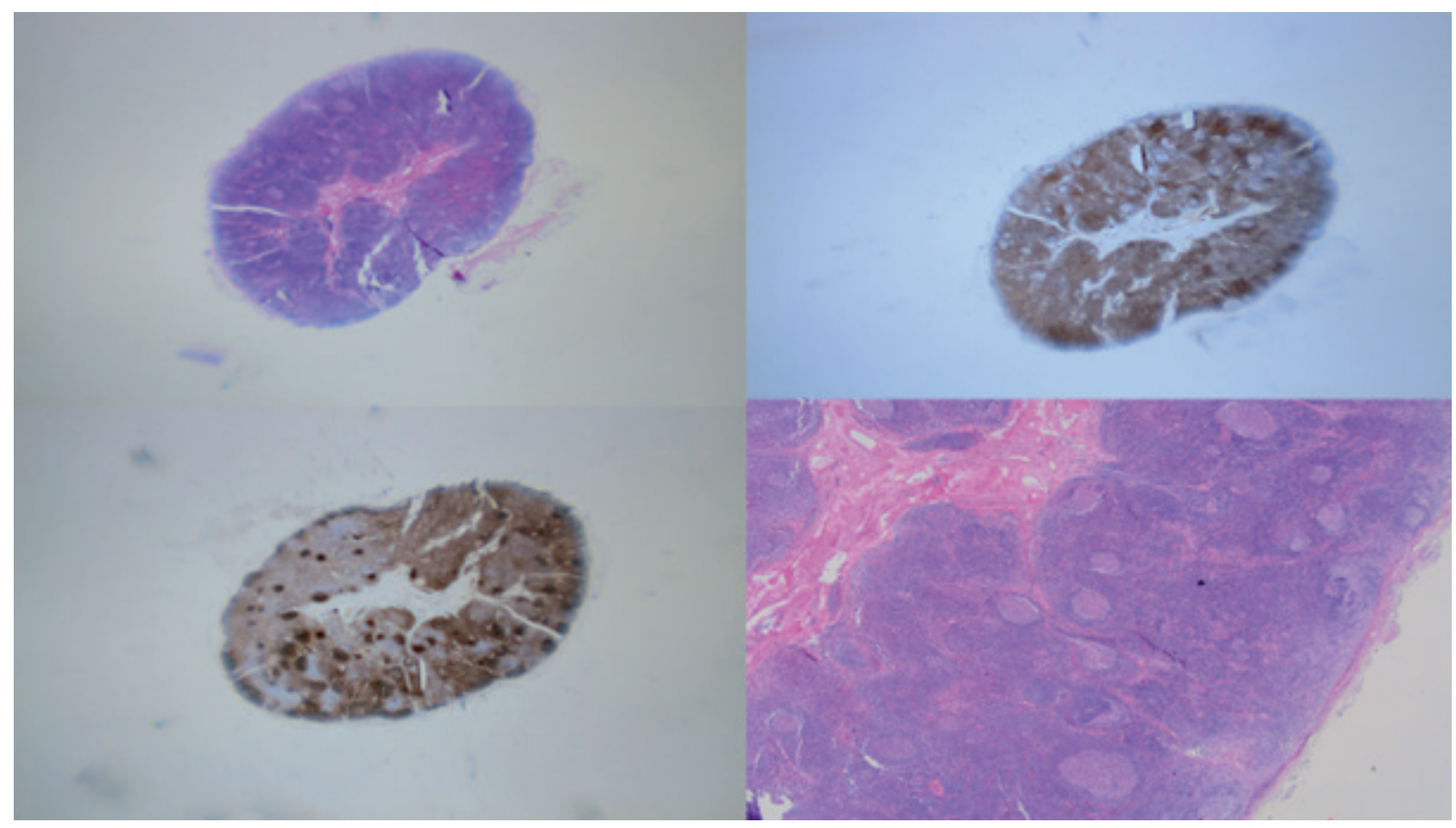

Figure 3. Low magnification showing preserved architecture with CD3 and CD20. Reactive germinal centers can be seen. There is an increased interfollicular B cell population.

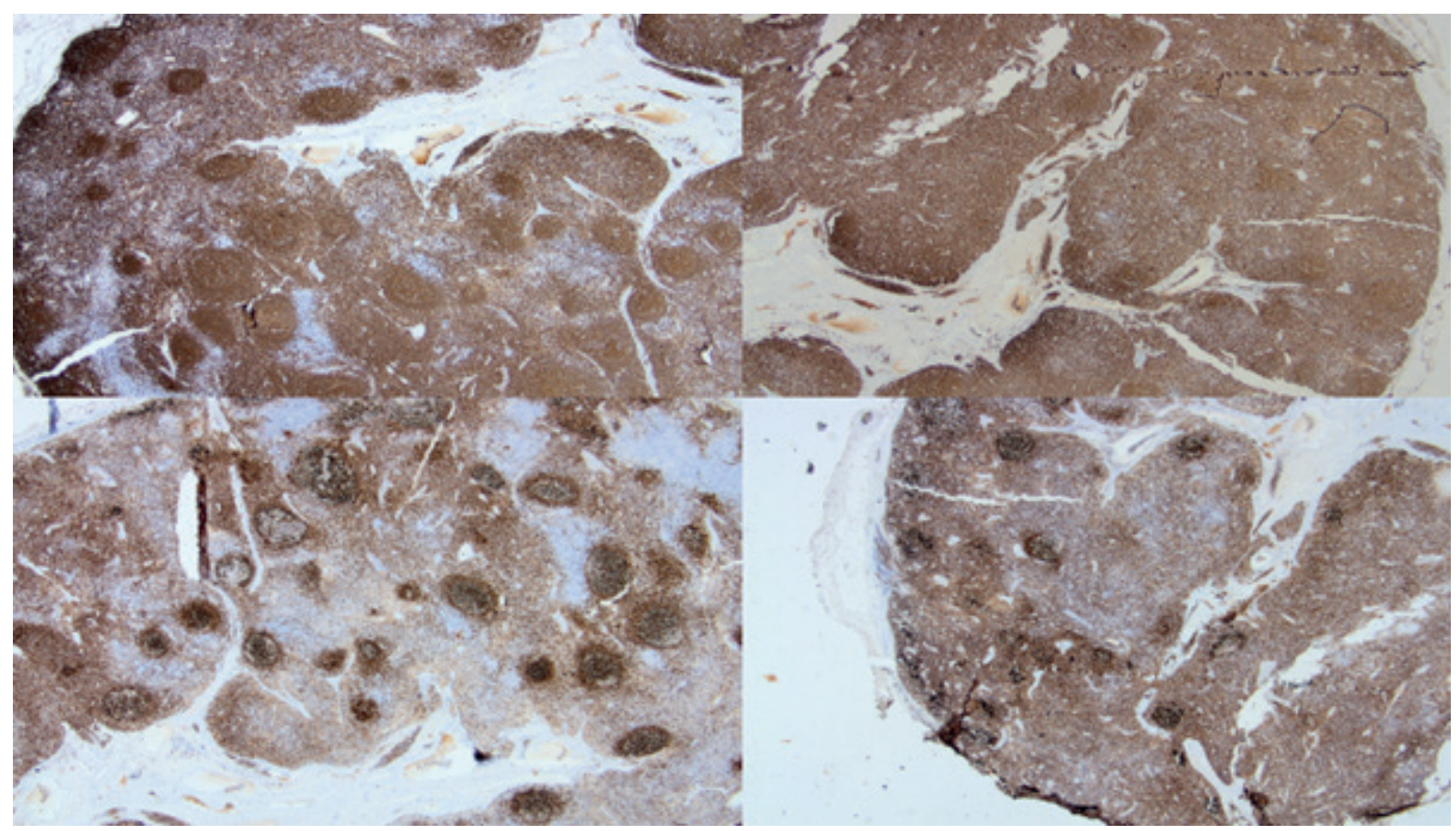

Figure 4. Close up showing an increased interfollicular B cell population. CD23 staining showing dendritic meshwork and increased marginal zone population. This patient was suspicious for marginal zone lymphoma but findings fell short of diagnosis.

\section{DISCUSSION}

Atypical lymphoproliferative disorders have significant potential for a malignant phenotype. This study showed that of 38 patients (73.1\%) who had
ALP in initial bone marrow or lymph node biopsy, were diagnosed with a hematologic malignancy or primary immunodeficiency during follow up. The complaints of the patients at the admission to the hematology clinic who had ALP in bone marrow 
or lymph node biopsy were lymphadenopathy, B symptoms, cytopenia (anemia, leukopenia, thrombocytopenia, bi-cytopenia or pancytopenia) and hepatosplenomegaly. These patients had been followed for median 9.2 months (0.0386.2). Thirty-eight patients were found to have a malignant lymphoproliferative disease or primary immunodeficiency disease during a follow-up that varied from 0.2-25 months. These patients were diagnosed with bone marrow (18.8\%), lymph node (68.8\%) biopsy or spleen (12.4\%) tissue. The primary immunodeficiency patients were diagnosed with immunoglobulin levels, their disease history and physical examination. Patients who developed hematological malignancy were given appropriate treatment regimen. Fourteen patients (26.9\%) did not develop any malignant lymphoproliferative disease during the follow up.

A study showed that of the 543 patients referred by their primary care doctors for further evaluation of lymphadenopathy, only $17.5 \%$ had an underlying neoplastic disease, with $11.4 \%$ having a lymphoproliferative disorder and $6.1 \%$ having a metastatic solid tumor. Thirty-one percent of the patients had benign reactive lymphadenopathy and $26 \%$ of the patients had non-neoplastic disease [3]. ALP in bone marrow or lymph node however, particularly if associated with hepatosplenomegaly, an abnormal complete blood count, or B symptoms, is particularly concerning for lymphoma or a systemic disease. An increased incidence of lymphoproliferative disease is observed in patients with primary immunodeficiencies [4]. The diagnosis of EBV associated lymphoproliferative disease can be difficult in immunodeficiency patients, who frequently have reactive lymphoid hyperplasia [2]. EBV infection is associated with a clinical spectrum of lymphoproliferative disease ranging from infectious mononucleosis to polyclonal post-transplant lymphoproliferation to Hodgkin lymphoma and non-Hodgkin lymphoma $[5,6]$.

This study had some limitations. First of all the study was retrospective. There were few patients in this study. Further studies are necessary in order to conclude ALP could predict a hematologic disease in the follow up. Until data of prospective studies are available, patients with ALP should be followed up in hematology out-patient clinic with caution for developing hematologic neoplastic disease. Additionally, each patient with atypical lymphoproliferation should be evaluated in terms of primary immunodeficiency.

In conclusion, hematological neoplasia was diagnosed in $32(61.6 \%)$ of the 52 patients and primary immunodeficiency developed in 6 (11.5\%) of the 52 patients. Patients who have ALP in lymph node or bone marrow biopsy should be followed up in the hematology outpatient clinic. Because, during follow-up, diseases such as hematological neoplasia or immunodeficiency can be diagnosed in patients with ALP.

\section{CONFLICT of INTERESTS}

The authors of this paper have no conflict of interests, including specific financial interests, relationships, and/or affiliations relevant to the subject matter or materials included.

\section{REFERENCES CQen}

[1] Schroer KR, Franssila KO. Atypical hyperplasia of lymph nodes A follow-up study. Cancer. 1979; 44(3): 1155-63.

[2] Greiner T, Armitage JO, Gross TG. Atypical lymphoproliferative diseases. ASH Education Program Book. 2000; 2000(1): 133-46.

[3] Chau I, Kelleher M, Cunningham D, et al. Rapid access multidisciplinary lymph node diagnostic clinic: analysis of 550 patients. British Journal of Cancer. 2003; 88(3): 354-61.

[4] Filipovich A, Jyonouchi $H$, Gross $T$, et al. Hematologic and oncologic complications of primary and secondary immunodeficiencies, including EBV related disorders. Immunologic disorders of infants and children, 4th ed Philadelphia, PA: Saunders. 1996; 855-88.

[5] Seiden MV, Sklar J. Molecular genetic analysis of posttransplant lymphoproliferative disorders. Hematology/Oncology Clinics. 1993; 7(2): 447-65.

[6] Shibata D, Weiss LM, Nathwani BN, et al. Epstein-Barr virus in benign lymph node biopsies from individuals infected with the human immunodeficiency virus is associated with concurrent or subsequent development of nonHodgkin's lymphoma. Blood. 1991; 77(7): 1527-33. 